\title{
INFANTILE SUPRACONDYLAR FRACTURE
}

\author{
A. L. Macafee, Belfast, Northern Ireland \\ From the Royal Belfast Hospital for Sick Children
}

Supracondylar fracture of the humerus is common in childhood but rare in infancy. Evidence is presented, illustrated in three cases, of the diagnostic difficulties encountered when this lesion is present in the infant.

Case 1-A girl aged nineteen months was first seen in May 1965, having fallen and injured her left arm. Elbow movements were painful and limited and the area was swollen. Radiographs (Fig. 1) at first suggested a dislocation but further films (Fig. 2) the following day, and manipulation under general anaesthesia, showed that the lesion was an unstable supracondylar fracture, which could be reduced by lateral pressure. A collar-and-cuff support was supplied. Recovery was satisfactory and two years later a full range of movement ( 0 to 140 degrees) was present. There was no deformity and the radiographs were normal.

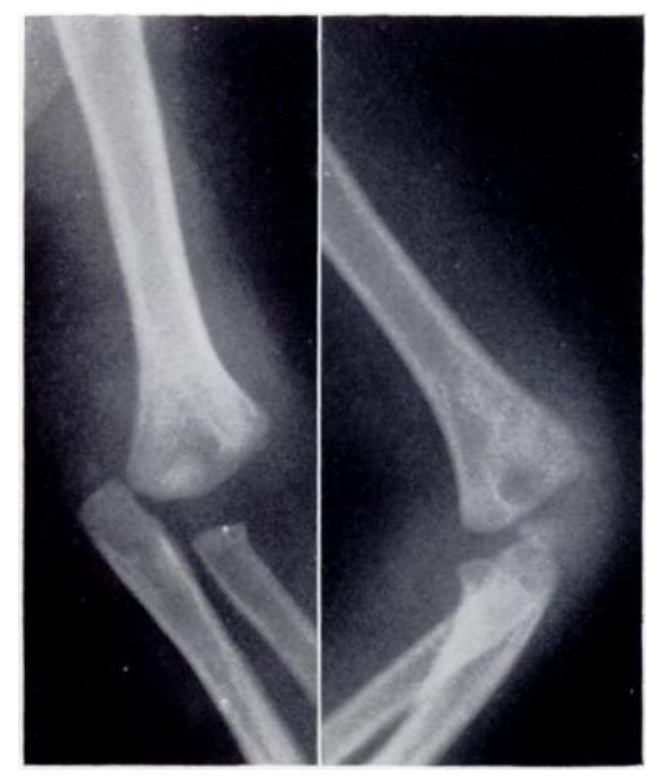

Fig. 1

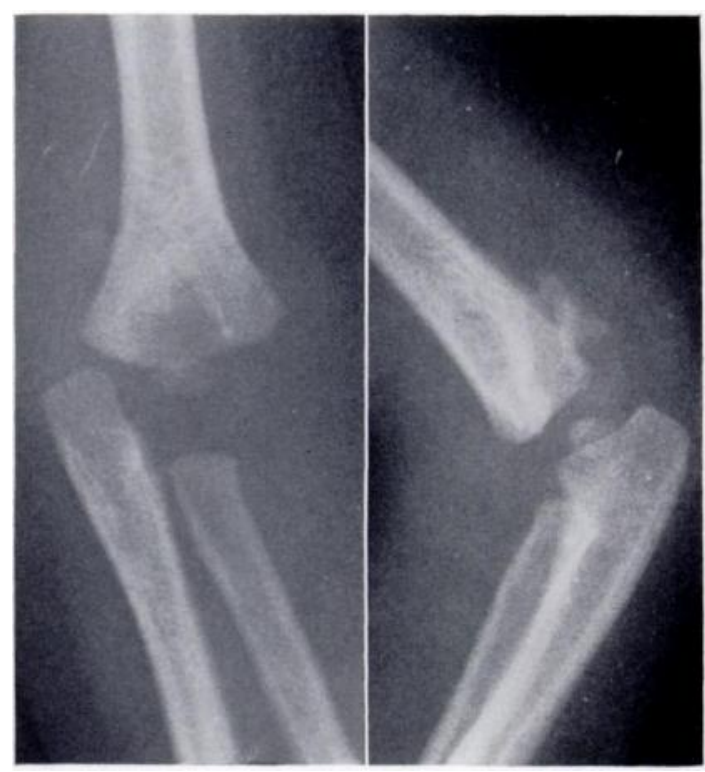

Fig. 2

Case 1. Figure 1-Initial radiographs suggesting a simple dislocation. Figure 2-Radiographs taken in theatre showing the supracondylar fracture.

Case 2-A boy aged twelve months was seen in March 1966 the day after a fall on his left arm. The elbow was swollen and movements were both painful and limited. Radiographs suggested initially a dislocation of the joint in addition to a fracture of the radius (Fig. 3). Reduction under general anaesthesia was not successful and the lesion remained unstable. The limb was splinted (Fig. 4). One week later exploration was performed through an anterior incision, when it was found that the lesion was not a dislocation but an unstable supracondylar fracture. The fragments were transfixed by a Kirschner wire and the limb was splinted 
(Fig. 5). Three weeks later the wire was removed; some infection was noted along the tract and a small sinus later required curettage. By twelve weeks the wound had healed and joint range was from 30 to 100 degrees.

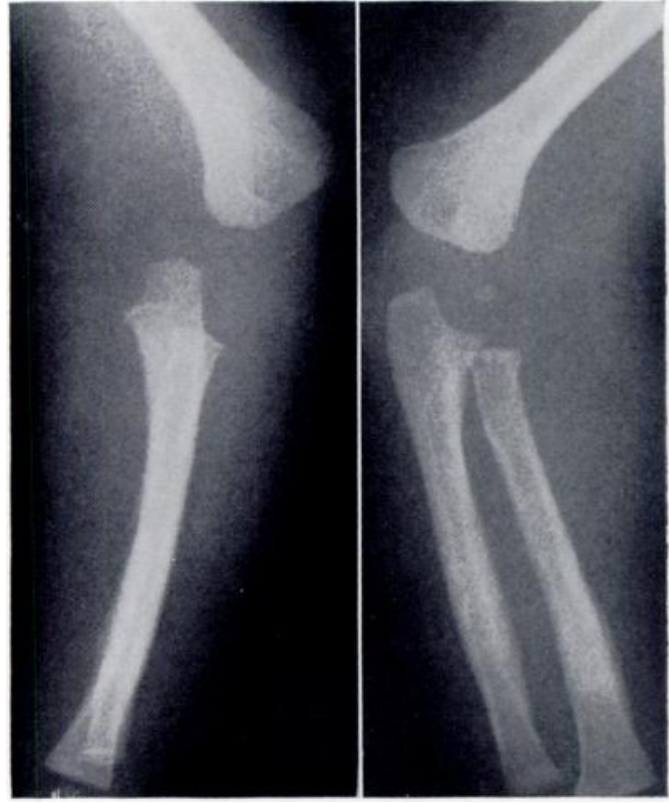

Fig. 3

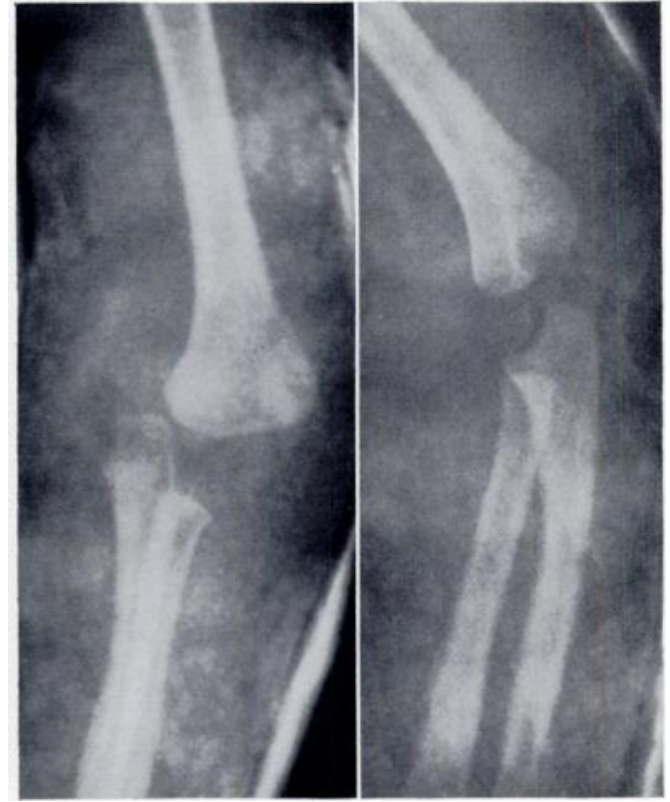

Fig. 4

Case 2. Figure 3-Suspected dislocation of elbow before manipulation. Callus formation at lower end of radius suggesting a previous fracture. Figure 4-Position in plaster after unsuccessful manipulation.

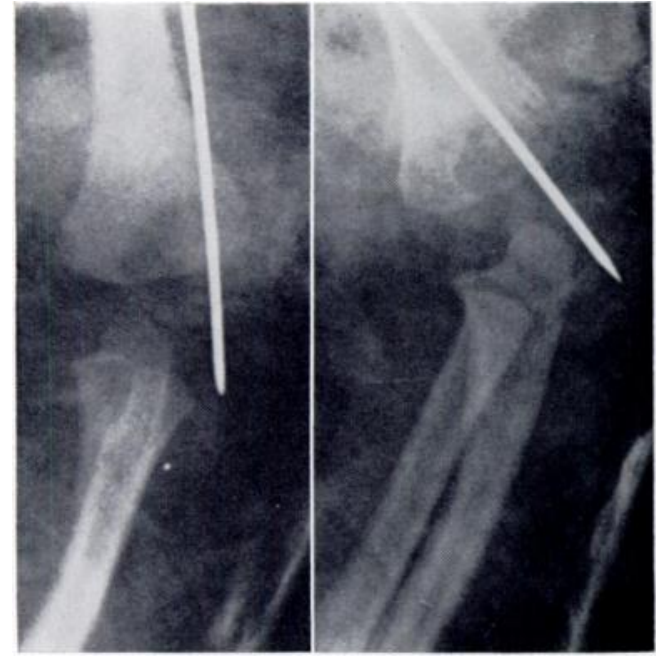

Fig. 5

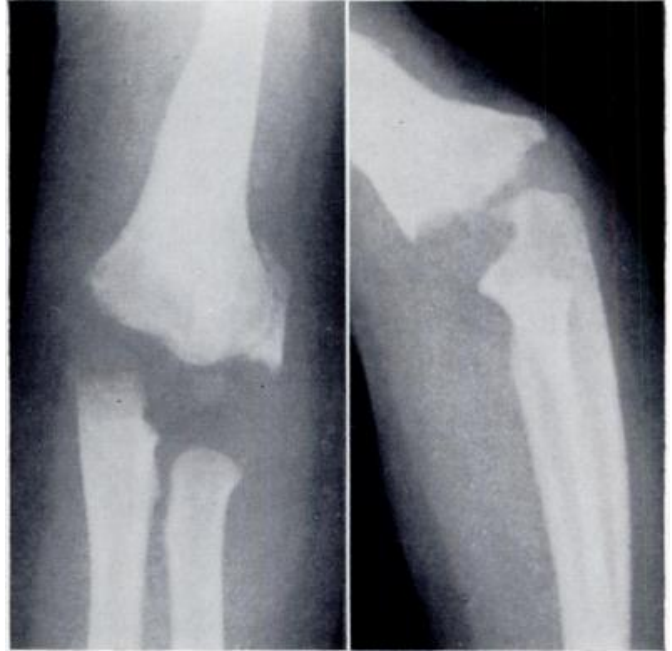

FIG. 6

Case 2. Figure 5-After internal fixation of fracture by a Kirschner wire. Figure 6-Review radiographs showing the old fracture at the lower end of the humerus.

Radiographs six months after the injury showed evidence of old fracture at the lower end of the left humerus (Fig. 6), and joint range a year after the injury was from 30 to 115 degrees. This infant has also been attending another hospital with a diagnosis of " battered baby syndrome."

VOL. 49 B, NO. 4, NOVEMBER 1967 


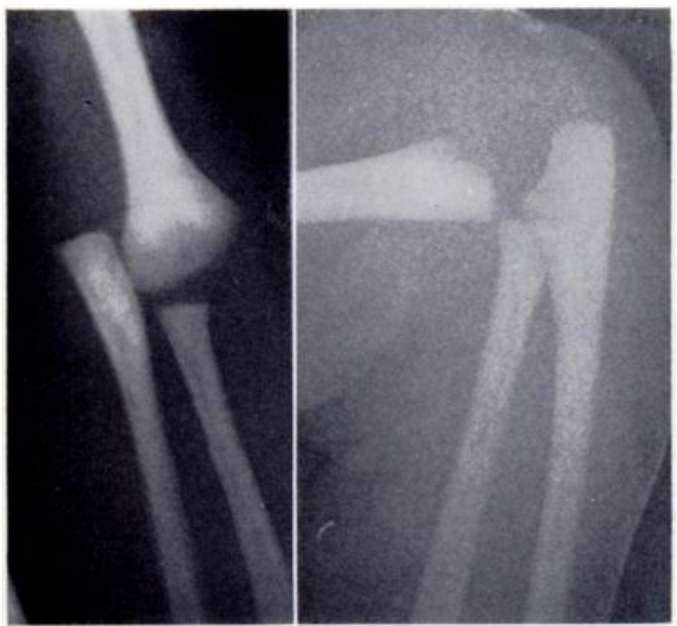

FIG. 7

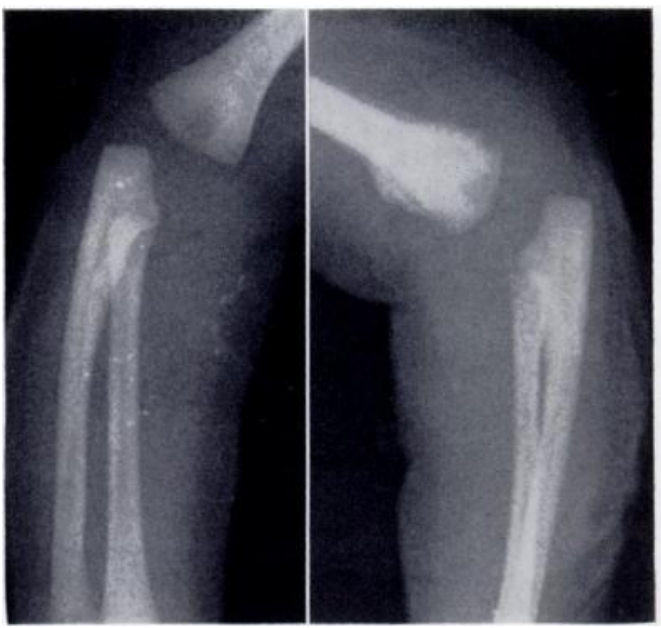

FIG. 8

Case 3. Figure 7-Suspected dislocation of left elbow joint. Figure 8-Callus formation at the lower end of the humerus at the age of 13 days.

Case 3-A boy aged two days was referred to the hospital in August 1966 because he was not using his left arm. His mother had noticed the previous day that there was tenderness in the left elbow. Initial radiographs suggested a dislocation of the left elbow (Fig. 7). Two

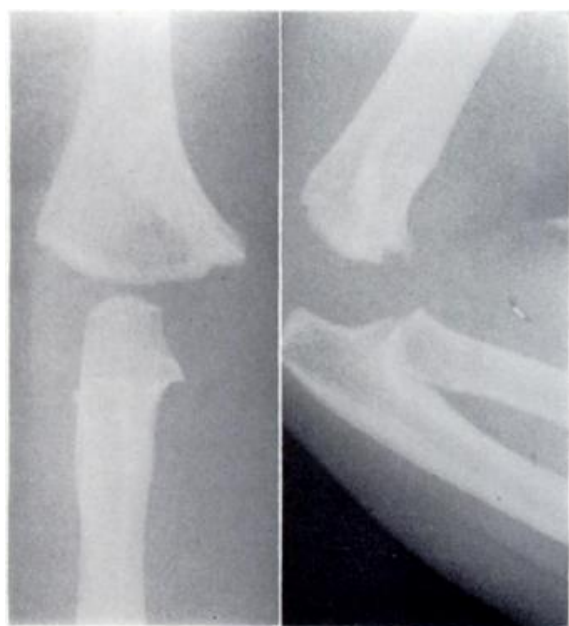

Fig. 9

Case 3-Review radiographs at the age of 9 months. The joint was clinically normal. attempts at reduction were made, the second under general anaesthesia; both were considered unsatisfactory. Orthopaedic opinion favoured a supracondylar fracture. Further radiographs eleven days after the injury showed profuse callus formation, suggesting that the injury had been a supracondylar fracture through the cartilaginous epiphysial area of the humerus rather than a dislocation (Fig. 8). The elbow was supported in a sling and a full range of elbow movements was regained within five weeks. At review nine months after the injury radiographs showed no significant abnormality (Fig. 9).

Comment-In all these cases dislocation of the elbow had been suspected at first on clinical and radiological grounds. Instability after manipulation led in two cases to the diagnosis of supracondylar fracture, which was treated conservatively. In the other case the diagnosis was made at open operation.

In the first two cases there was associated bruising, but there was no evidence of underlying disease to account for the apparent proclivity to haematoma formation. The third fracture is considered to have been a birth injury.

\section{SUMMARY}

Three cases are described of elbow injuries in infants, to illustrate the difficulty of differentiating a dislocation from a supracondylar fracture.

I wish to thank Mr B. T. Crymble and Mr J. Piggot for permission to publish these cases. 